\title{
Supporting Information: Mining For Ligandable Cavities in RNA
}

Jingru Xie ${ }^{1}$ and Aaron T. Frank ${ }^{2,3^{*}}$

${ }^{1}$ University of Michigan, Physics, Ann Arbor, 48109, United States of America

${ }^{2}$ University of Michigan, Biophysics, Ann Arbor, 48109, United States of America

${ }^{3}$ University of Michigan, Chemistry, Ann Arbor, 48109, United States of America

*afrankz@umich.edu

\section{Contents}

1 Ligandability Classifiers $\quad \mathbf{S 2}$

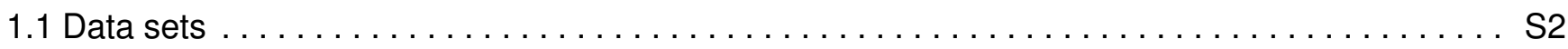

1.2 Receiver-operator-characteristic $(\mathrm{ROC})$ Analysis $\ldots \ldots \ldots \ldots \ldots \ldots \ldots \ldots \ldots \ldots \ldots \ldots . \ldots \ldots$

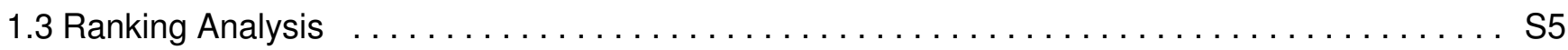

2 Mining for Ligandable Pockets in HIV-1 TAR $\quad$ S8

2.1 3D Structure Prediction $\ldots \ldots \ldots \ldots \ldots \ldots \ldots \ldots \ldots \ldots \ldots \ldots \ldots \ldots \ldots \ldots \ldots \ldots \ldots$

2.2 Grid-based visualization of ligandable sites in top six structures $\ldots \ldots \ldots \ldots \ldots \ldots \ldots \ldots$ S9

2.3 Virtual Screening Assessment $\ldots \ldots \ldots \ldots \ldots \ldots \ldots \ldots \ldots \ldots \ldots \ldots \ldots \ldots \ldots \ldots \ldots$

2.4 Docking comparison between the top 20 and bottom 20 SimRNA structures . . . . . . . . S12 


\section{Ligandability Classifiers}

\subsection{Data sets}

\begin{tabular}{ll}
\hline Dataset & PDB IDs \\
\hline Training (88) & 1F27 1FUF 1J7T 1LC4 1MWL 1NTA 1NTB 1O9M 1U8D 1YRJ 1ZZ5 \\
& 2BE0 2BEE 2CKY 2EEU 2EEV 2EEW 2ET3 2ET4 2ET5 2ET8 2F4S \\
& 2F4T 2F4U 2FCX 2FCY 2FCZ 2G5Q 2HOJ 2HOM 2HOO 2O3V 2O3W \\
& 2O3X 2O3Y 2OE5 2OE8 2QWY 3B4B 3B4C 3C44 3D2G 3D2V 3D2X \\
& 3E5E 3F2Q 3F2T 3F4H 3GX2 3GX3 3GX5 3GX6 3GX7 3IQN 3IQR \\
& 3NPQ 3SUH 3TZR 3WRU 4B5R 4F8U 4F8V 4FAW 4GPW 4GPX 4GPY \\
& 4K32 4L81 4LVX 4LVY 4LW0 4P20 4P3S 4PDQ 4QLM 4QLN 4TS2 \\
& 4TZX 4TZY 4WCR 4YAZ 4ZNP 5BTP 5BWS 5BXK 5C45 5KX9 6BFB \\
\hline Test Set 1 (X-ray) (23) & 1F1T 2B57 2G5K 2XNW 2YDH 3FU2 3LA5 3MUM 3NPN 3Q50 3SD3 \\
& 3SLM 4AOB 4ERJ 4FE5 4JF2 4KQY 4LX5 4NYA 4XWF 4YB0 5C7W \\
& 5KPY \\
\hline Test Set 2 (NMR) (20) & 1AJU 1AKX 1AM0 1BYJ 1EHT 1EI2 1EVV 1FMN 1KOC 1KOD 1LVJ \\
& 1NEM 1PBR 1Q8N 1QD3 1TOB 1UTS 1UUD 1UUI 2TOB \\
\hline
\end{tabular}

Table S1. PDB IDs for systems used to train and test our ligandability classifiers. A detailed summary of this data is available via https://github.com/atfrank/RNACavityMiner/blob/master/data_summary/. Cavity data used to training and test the classifiers were mapped using rbcavity from the rDock modeling suite.[1]

To be able to separate "ligandable" cavities from decoys, we trained a set of five machine learning classification models: an extreme gradient boosting (XGB), a random forest (RF), a multilayer perceptron (MLP, also referred to as a feed-forward artificial neural network), a logistic regression (LR), and an extra-randomized trees (ERT) classifier. All the classifiers take in the cavity FP and output the "ligandability" score. The ligandability score is a number ranging from zero to one that represents an estimate of the likelihood that the cavity associated with a cavity FP is "ligandable". If the score is closer to one, it indicates the associated cavity has a high probability being "ligandable," while a score closer to zero indicates the associated cavity is likely a decoy. A consensus classifier was also used. To test the five classifier we applied it to two independent test sets. We also tested a consensus classifier, which we defined as the mean predictions from the XGB, RF, MLP, LR, and ERT classifiers. In Figure S2 results as function of the distance threshold used to determine classification success. In Table S2 and S2 we summarized the results for each RNA in test set 1 and 2, respectively when utilizing the consensus classifier and distance threshold of $6.0 \AA$. 


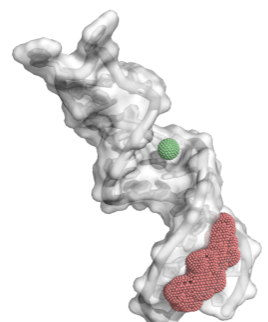

$1 \mathrm{AJU}$

(Distance $20.8 \AA$ )
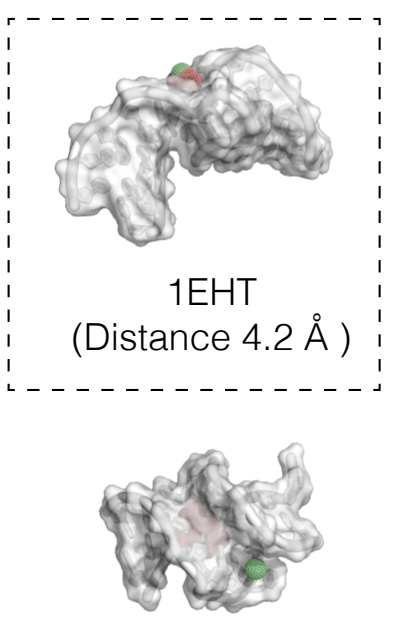

$1 \mathrm{KOD}$

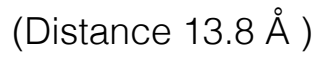

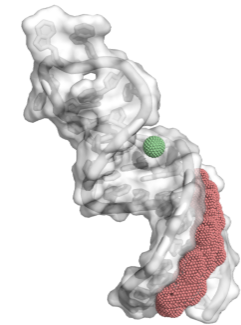

$1 \mathrm{AKX}$
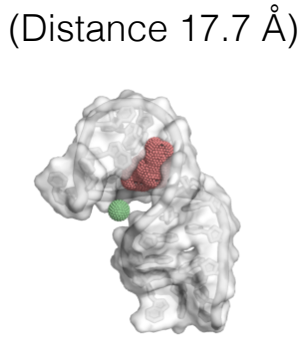

$1 \mathrm{EI} 2$

(Distance $9.7 \AA$ )
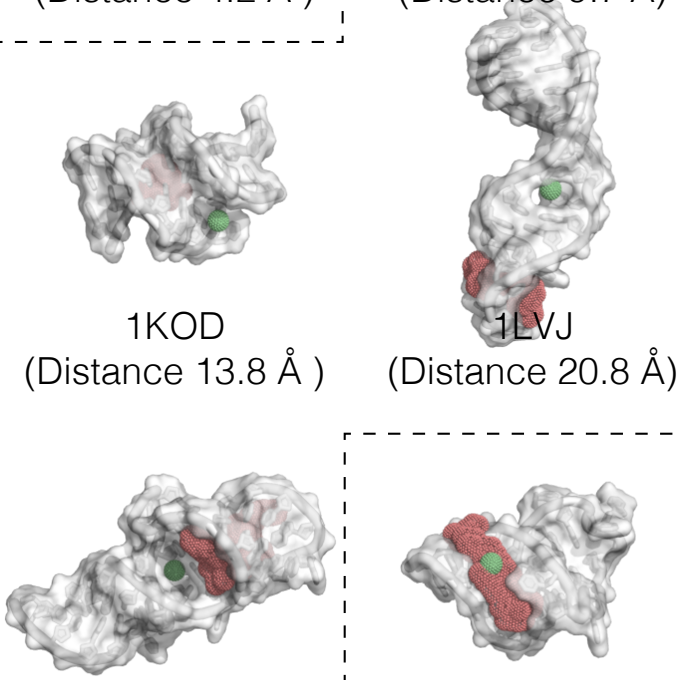

1Q8N

(Distance 11.4 ̊ )
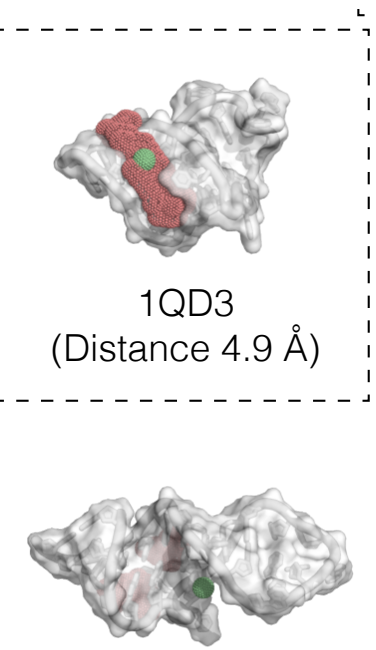

1 UUI

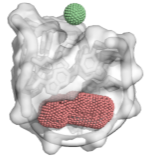

1AM0

(Distance $15.9 \AA$ )

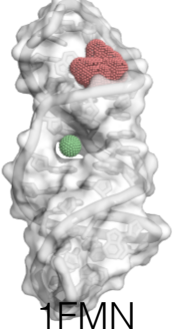

(Distance $15.2 \AA$ )

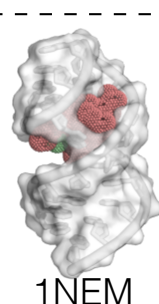

(Distance $5.5 \AA$ )

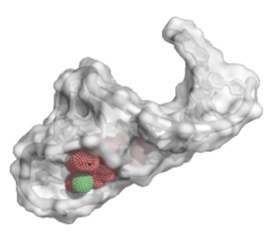

1TOB

(Distance $7.0 \AA$ )

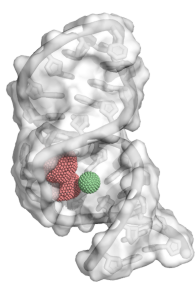

1BYJ

(Distance $6.6 \AA ̊$ )

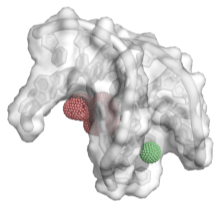

$1 \mathrm{KOC}$

(Distance $11.0 \AA$ )

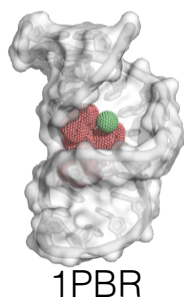

(Distance $7.3 \AA$ )

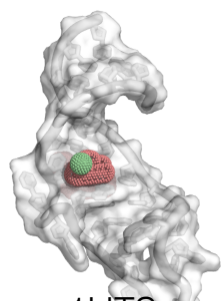

1 UTS

(Distance $9.5 \AA$ )

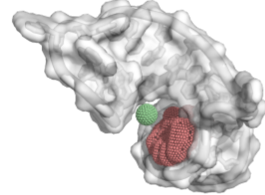

1UUD

\section{(Distance $11.6 \AA$ )}

(Distance $13.4 \AA$ )

Figure S1. Binding sites identified in test set 2 using the program, autosite[2] (salmon red spheres). For reference, we show the center of the native bind cavity as green spheres. The centroid distance between the binding site identified by autosite and the native cavity is indicated under each figure. Boxes highlight the instances where this distance is less than $6.0 \AA$. 


\subsection{Receiver-operator-characteristic (ROC) Analysis}
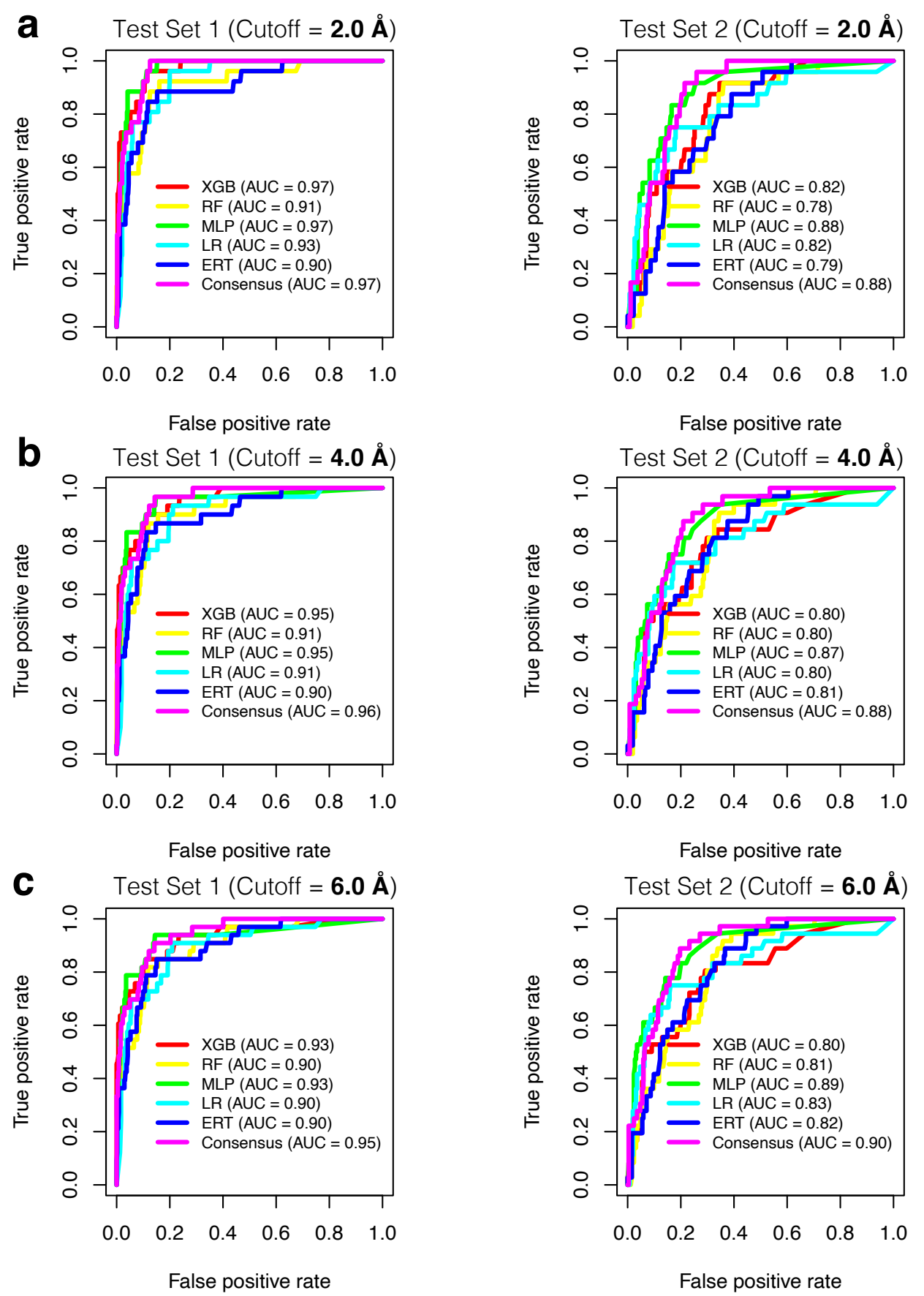

Figure S2. Shown in this figure is the ROC analysis as a function of cutoff used to define success. Shown are results when we define classification as successful if the pseudoatom with the highest ligandability score is (a) 2.0, (b) 4.0, and (c) $6.0 \AA$ within the center of the native cavity. 


\subsection{Ranking Analysis}

\begin{tabular}{|c|c|c|c|c|c|}
\hline PDB & Description & nts & QED & FP Dissimilarity & Rank \\
\hline 1F1T & Malachite green RNA aptamer & 38 & 0.739 & 8.213 & $1(19)$ \\
\hline 2B57 & C74U mutant Guanine riboswitch & 65 & 0.470 & 1.475 & $1(15)$ \\
\hline 2G5K & Ribosomal decoding site (A-site) & 20 & 0.149 & 2.398 & $2(5)$ \\
\hline 2XNW & Guanine riboswitch & 64 & 0.208 & 2.152 & $1(21)$ \\
\hline 2YDH & SAM-I riboswitch & 94 & 0.282 & 0.357 & $2(29)$ \\
\hline 3FU2 & PreQ1 riboswitch & 31 & 0.463 & 5.818 & $1(14)$ \\
\hline 3LA5 & Adenosine riboswitch & 71 & 0.439 & 1.963 & $1(24)$ \\
\hline 3MUM & G20A mutant c-di-GMP riboswith & 92 & 0.120 & 5.297 & $2(16)$ \\
\hline $3 N P N$ & SAM riboswitch & 50 & 0.224 & 0.990 & $4(18)$ \\
\hline 3Q50 & PreQ1 riboswitch & 33 & 0.463 & 5.841 & $1(13)$ \\
\hline 3SD3 & Tetrahydrofolate riboswitch & 89 & 0.540 & 4.273 & $6(22)$ \\
\hline $3 S L M$ & 2'- Deoxyguanosine riboswitch & 68 & 0.268 & 6.381 & $4(28)$ \\
\hline $4 A O B$ & SAM-I riboswitch & 94 & 0.282 & 0.699 & $1(28)$ \\
\hline 4ERJ & Lysine riboswitch RNA & 161 & 0.360 & 11.402 & $1(46)$ \\
\hline 4FE5 & xpt-pbuX guanine riboswitch & 67 & 0.519 & 0.555 & $1(20)$ \\
\hline 4JF2 & PreQ1-II riboswitch & 77 & 0.463 & 8.221 & $2(27)$ \\
\hline $4 K Q Y$ & YitJ S box/SAM-I riboswitch & 119 & 0.282 & 2.924 & $1(28)$ \\
\hline $4 \mathrm{LX} 5$ & Mutated adenine riboswitch & 71 & 0.545 & 2.321 & $1(20)$ \\
\hline 4NYA & thiM TPP riboswitch & 80 & 0.335 & 2.549 & $1(27)$ \\
\hline 4XWF & ZMP riboswitch & 64 & 0.337 & 3.485 & $3(26)$ \\
\hline 4YB0 & 3',3'-cGAMP riboswitch & 83 & 0.120 & 1.040 & $2(23)$ \\
\hline $5 \mathrm{C} 7 \mathrm{~W}$ & 5'-monophosphate Z:P guanine riboswitch & 65 & 0.519 & 1.003 & $1(25)$ \\
\hline \multirow[t]{2}{*}{$5 \mathrm{KPY}$} & 5-hydroxytryptophan RNA aptamer & 71 & 0.474 & 7.481 & $2(24)$ \\
\hline & mean & 72 & 0.374 & 3.76 & $1.8(22.5)$ \\
\hline
\end{tabular}

Table S2. Results of the cavity prediction for each of the systems in the X-ray test set. Values in the Rank column represent the rank position of the native cavity over the total number of cavities identified by rbcavity. Also listed are descriptions of the RNAs, their length, and the QED (drug-likeness) scores of the ligands. For reference, we also list for each test system the lowest FP dissimilarity relative to the data in the training set. 


\begin{tabular}{|c|c|c|c|c|c|}
\hline PDB & Description & nts & QED & FP Dissimilarity & Rank \\
\hline $1 \mathrm{AJU}$ & HIV-2 TAR (stem-loop) & 30 & 0.142 & 3.633 & $1(14)$ \\
\hline $1 \mathrm{AKX}$ & HIV-2 TAR (stem-loop) & 30 & 0.226 & 3.132 & $3(21)$ \\
\hline 1AM0 & AMP RNA aptamer (stem-loop) & 16 & 0.474 & 4.311 & $2(9)$ \\
\hline 1BYJ & Ribosomal decoding site (A-site stem-loop) & 27 & 0.193 & 1.284 & $1(11)$ \\
\hline 1EHT & Theophylline-binding RNA (stem-loop) & 33 & 0.562 & 5.582 & $2(18)$ \\
\hline 1EI2 & Tau exon 10 RNA (stem-loop) & 25 & 0.114 & 4.872 & $2(14)$ \\
\hline $1 \mathrm{EVV}$ & transfer RNA & 62 & 0.345 & 4.185 & $2(21)$ \\
\hline 1FMN & RNA aptamer (stem-loop) & 35 & 0.246 & 5.076 & $2(15)$ \\
\hline $1 \mathrm{KOC}$ & RNA aptamer (stem-loop) & 25 & 0.189 & 3.360 & $1(12)$ \\
\hline $1 \mathrm{KOD}$ & RNA aptamer (stem-loop) & 25 & 0.381 & 5.702 & $6(11)$ \\
\hline $1 \mathrm{LVJ}$ & HIV-1 TAR (stem-loop) & 31 & 0.855 & 7.177 & $2(18)$ \\
\hline 1NEM & Neomycin B RNA aptamer (stem-loop) & 23 & 0.114 & 4.591 & $2(11)$ \\
\hline 1PBR & Ribosomal decoding site (A-site stem-loop) & 27 & 0.114 & 1.660 & $1(16)$ \\
\hline 1Q8N & Malachite green RNA aptamer (stem-loop) & 38 & 0.608 & 7.091 & $1(23)$ \\
\hline 1QD3 & HIV-1 TAR (stem-loop) & 29 & 0.114 & 4.540 & $4(8)$ \\
\hline $1 \mathrm{TOB}$ & RNA aptamer (stem-loop) & 27 & 0.175 & 5.531 & $1(14)$ \\
\hline 1UTS & HIV-1 TAR (stem-loop) & 29 & 0.389 & 4.032 & $5(14)$ \\
\hline 1UUD & HIV-1 TAR (stem-loop) & 29 & 0.156 & 3.260 & $2(11)$ \\
\hline 1UUI & HIV-1 TAR (stem-loop) & 29 & 0.447 & 3.694 & $2(19)$ \\
\hline \multirow[t]{2}{*}{ 2TOB } & Tobramycin RNA aptamer & 20 & 0.175 & 7.864 & $1(10)$ \\
\hline & mean & 30 & 0.300 & 4.53 & $2.1(14.5)$ \\
\hline
\end{tabular}

Table S3. Results of the cavity prediction for each of the systems in the NMR test set. Values in the Rank column represent the rank position of the native cavity over the total number of cavities identified by rbcavity from the rDock modeling suite.[1]. Also listed are descriptions of the RNAs, their length, and the QED (drug-likeness) scores of the ligands. QEDs were computed using RDKit[3]. For reference, we also list for each test system the lowest FP dissimilarity relative to the data in the training set. 


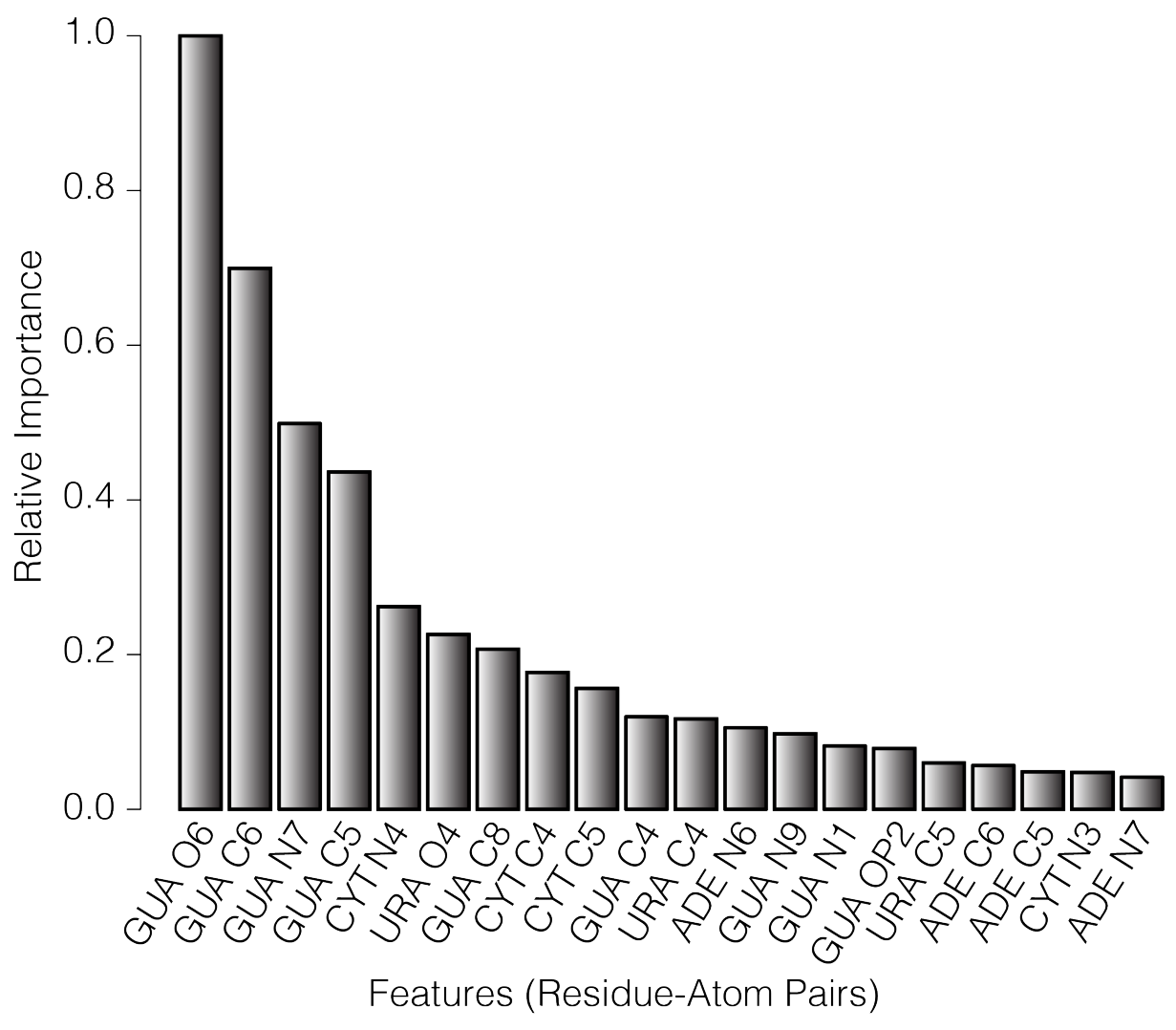

Figure S3. To determine which elements in our cavity FP are most important for classifying cavities as "ligandable", we carried out feature importance analysis using the feature importance scores of the random forest model. Because each element in the FP is associated with a specific residue-atom pair type in the RNA, this analysis allowed us to identify the most important residue-atom pair types. Shown in Figure $S 3$ is the plot of importance scores associated with the twenty most important pair types, defined by RNA residue types (ADE, GUA, CYT, and URA) and atom names. Most of the important features are associated with GUA residues and nucleobase atoms (Figure S3). Barplot of the relative importance scores. Shown are the ordered scores for the 20 importance features, aggregated by their residue-atom pairs. The importance scores were derived from the random forest model and their relative values indicate which features most impact the accuracy of the random forest classifier. Shown in the insets are rendering of the importance scores projected on the RNA-ligand system with PDB ID: 2 L94. Hotspot are shown in a orange-blue-white (most-to-least) color gradient. 


\section{Mining for Ligandable Pockets in HIV-1 TAR}

\subsection{D Structure Prediction}

To examine the utility of binding cavity classifiers to mine for bound-like structures, we carried out de novo modeling of the 27-nt transactivating response element (TAR) RNA, an RNA element found in the $5^{\prime}$-untranslated region (UTR) of the human immunodeficiency virus (HIV). We chose TAR because of its small size, and the availability of several holo structures (PDB ID: 1ARJ[4], 1QD3[5] 1UUD[6], 1UUI[6], 2L8H[7], 2KDQ[8], 2KX5[9], 2J0M[10], 5J1O[10], 5J2W[10], 6CMN[11]). We first generated structural models of TAR using the 3D RNA folding tool, SimRNA [12]. The target secondary structure we used for this folding simulation was taken from the apo structure of TAR (PDB ID: 1ANR)[13]. The folding simulation of TAR was run for $64,000,000$ steps and coordinates were saved to the trajectory every 10,000 steps. The initial simulated annealing temperature was set to 1.35 and the final temperature to 0.90 . The weights for the bond, angle, and $\eta_{\theta}$ terms in the coarse-grained SimRNA force field were set to 1.00,1.00, and 0.40 , respectively. For our simulation, we ran SimRNA in replica exchange Monte Carlo mode, with the number of replicas set to 10. The resulting trajectories from each replica were combined, and the collection of 64,000 structures was clustered with the maximum RMSD threshold set to $5.0 \AA$. For each resulting cluster, the structure with the lowest energy was selected. After applying the protocol described above, a total of 1455 low-energy structures were obtained. Each of the 1455 low-energy structures was further energy-minimized using the molecular mechanics modeling program CHARMM[14] and the CHARMM36 nucleic acid force field[15]. Next, for each of the energy-minimized structures, we carried out binding cavity analysis using the program rbcavity from the rDock modeling suite.[1] For each identified cavity, we a placed a pseudoatom at the center of the cavity, and then applied atomic fingerprinting to the pseudoatom (Eq. 1 (main text)) to derive a cavity FP. We then used our classifiers to score the ligandability of the cavities in the modeled TAR structures from their corresponding cavity FPs. The top 20 SimRNA TAR-conformers with the highest ligandability scores were selected and compared to known holo-TAR structures. 


\subsection{Grid-based visualization of ligandable sites in top six structures}
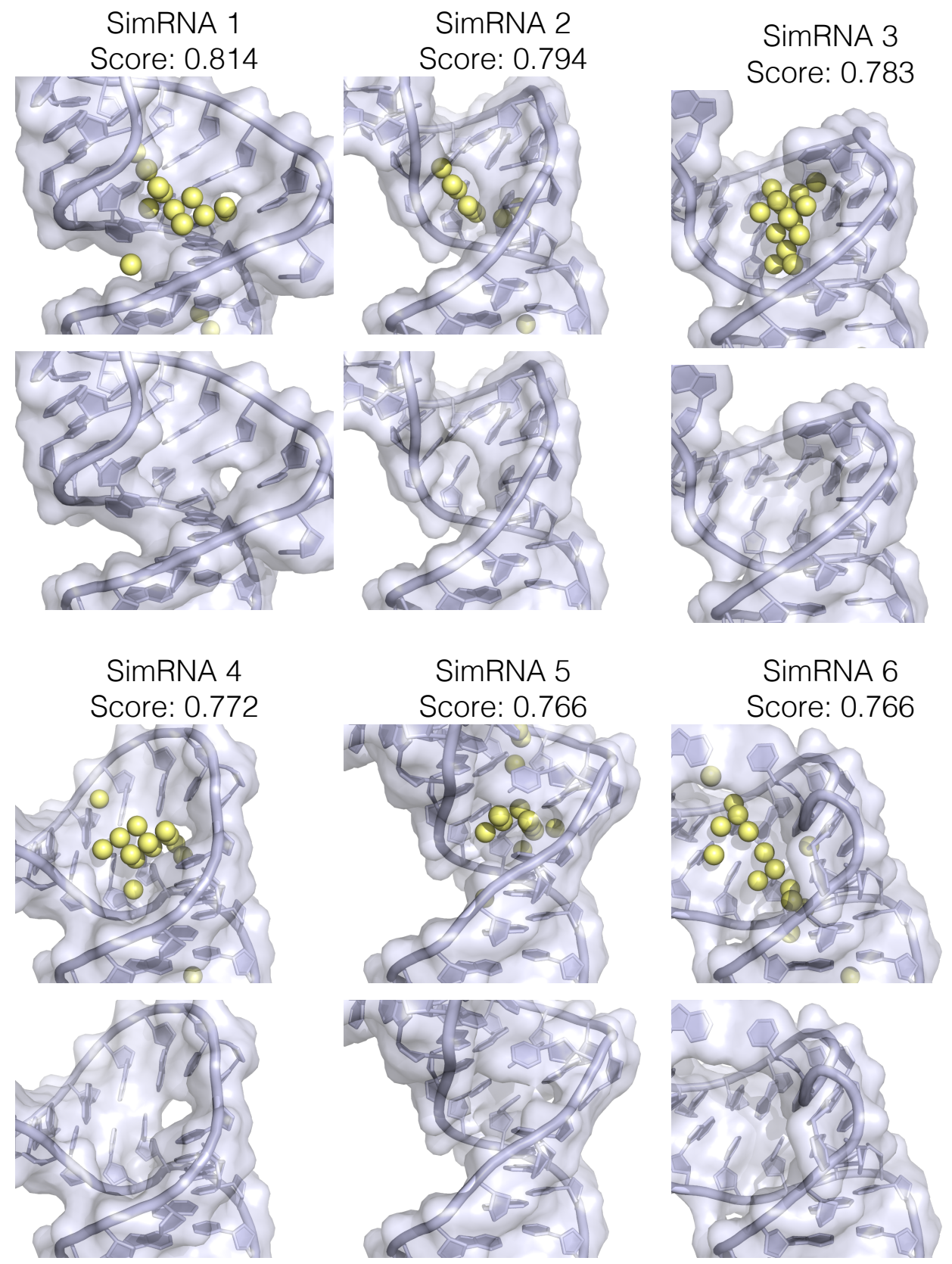

Figure S4. Shown are grid points on the surface of the TAR SimRNA structures that exhibited high $(>0.60)$ ligandability scores. Here the grid points are rendered as spheres. For reference, we show the structures without gridpoints at the bottom. High ligandability grid points tend to cluster near complex, structurally irregular regions in TAR.

\subsection{Virtual Screening Assessment}

We docked a library of small-molecule ligands to each of the 20 SimRNA TAR-conformers with the highest ligandability scores. The library contained 102326 small molecules, 26 of which were 


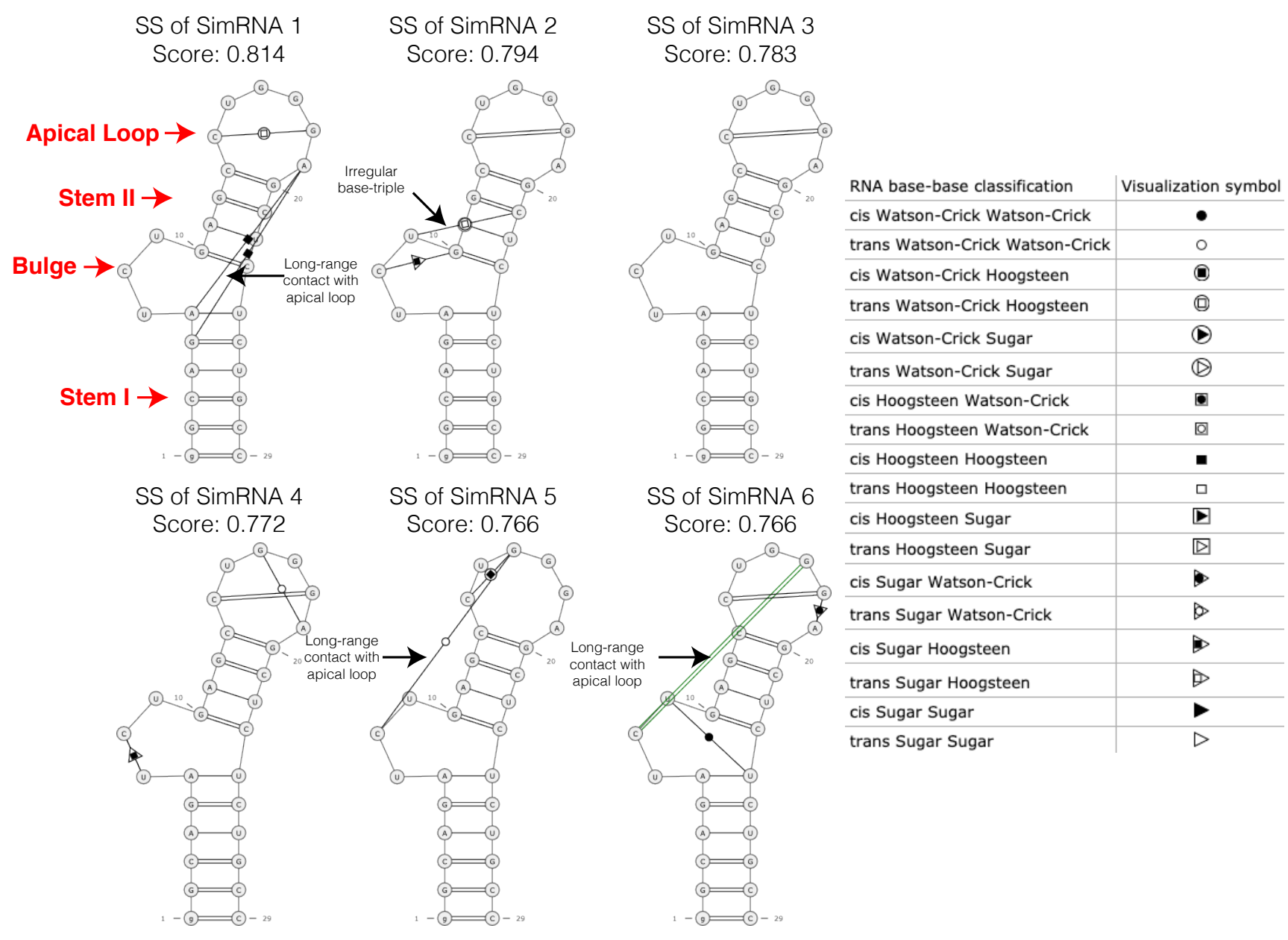

Figure S5. Secondary structure (SS) of the six SimRNA TAR models with the highest ligandability scores. The secondary structures were annotated from the 3D SimRNA structures using the RNApdbee webserver[16].

validated binders of HIV-1 TAR[17]. We used the program rDock[1], for our docking calculations. For each ligand and each conformer, we selected the best pose using the total interaction energy. We then calculated the ROC AUCs and 1\% and $2 \%$ enrichment factors (EF) when sorting the library based on the Boltzmann-averaged docking scores across the 20 conformers. That i.e., for given ligand $(i)$ in the library, the docking score

$$
\Delta G_{i}=\sum_{j} w_{i, j} \Delta G_{i, j}
$$

where $j$ runs over the conformers in ensemble (here the 20 SimRNA structures), $\Delta G_{i, j}$ is the rDock interaction score for ligand $i$ docked to conformer $j$, and $w_{i, j}$ is the Boltzmann weight, which is given as

$$
w_{i, j}=\frac{e^{-\Delta G_{i, j} / k_{B} T}}{\sum_{j} e^{-\Delta G_{i, j} / k_{B} T}}
$$

where $k_{B}$ and $T$ are the Boltzmann constant and temperature, respectively. 


\begin{tabular}{lcrr}
\hline & ROC AUC & 1\%EF & 2\% EF \\
\hline Ensemble & $\mathbf{0 . 8 4}$ & $\mathbf{2 3 . 1}$ & $\mathbf{2 1 . 1}$ \\
\hline SimRNA 1 & 0.79 & 15.4 & 7.7 \\
SimRNA 2 & 0.84 & 11.5 & 11.5 \\
SimRNA 3 & 0.79 & 19.2 & 11.5 \\
SimRNA 4 & 0.73 & $\mathbf{2 6 . 9}$ & 15.4 \\
SimRNA 5 & 0.82 & $\mathbf{2 6 . 9}$ & 13.5 \\
SimRNA 6 & 0.80 & 15.4 & 9.6 \\
SimRNA 7 & 0.76 & 19.2 & 13.5 \\
SimRNA 8 & 0.84 & $\mathbf{2 6 . 9}$ & $\mathbf{1 7 . 3}$ \\
SimRNA 9 & 0.79 & 19.2 & 13.4 \\
SimRNA 10 & 0.81 & 23.1 & 11.5 \\
SimRNA 11 & 0.84 & 3.8 & 13.4 \\
SimRNA 12 & 0.77 & 11.5 & 7.7 \\
SimRNA 13 & 0.77 & 15.4 & 11.5 \\
SimRNA 14 & 0.85 & 19.2 & 13.5 \\
SimRNA 15 & 0.76 & 7.7 & 3.8 \\
SimRNA 16 & $\mathbf{0 . 8 6}$ & 15.4 & 13.5 \\
SimRNA 17 & 0.73 & 19.2 & 11.5 \\
SimRNA 18 & 0.79 & 19.2 & 13.4 \\
SimRNA 19 & 0.82 & 11.5 & 9.6 \\
SimRNA 20 & 0.78 & 11.5 & 9.6 \\
\hline
\end{tabular}

Table S4. Ensemble-docking results against the 20 SimRNA structures that harbored the highest-scoring binding cavities. ROC AUC and $1 \%$ and $2 \%$ enrichment factors (EF) are listed when using the mean ensemble docking scores to sorted compounds in the screening library. Also listed are resulted based when using the individual docking scores. 


\subsection{Docking comparison between the top 20 and bottom 20 SimRNA structures}

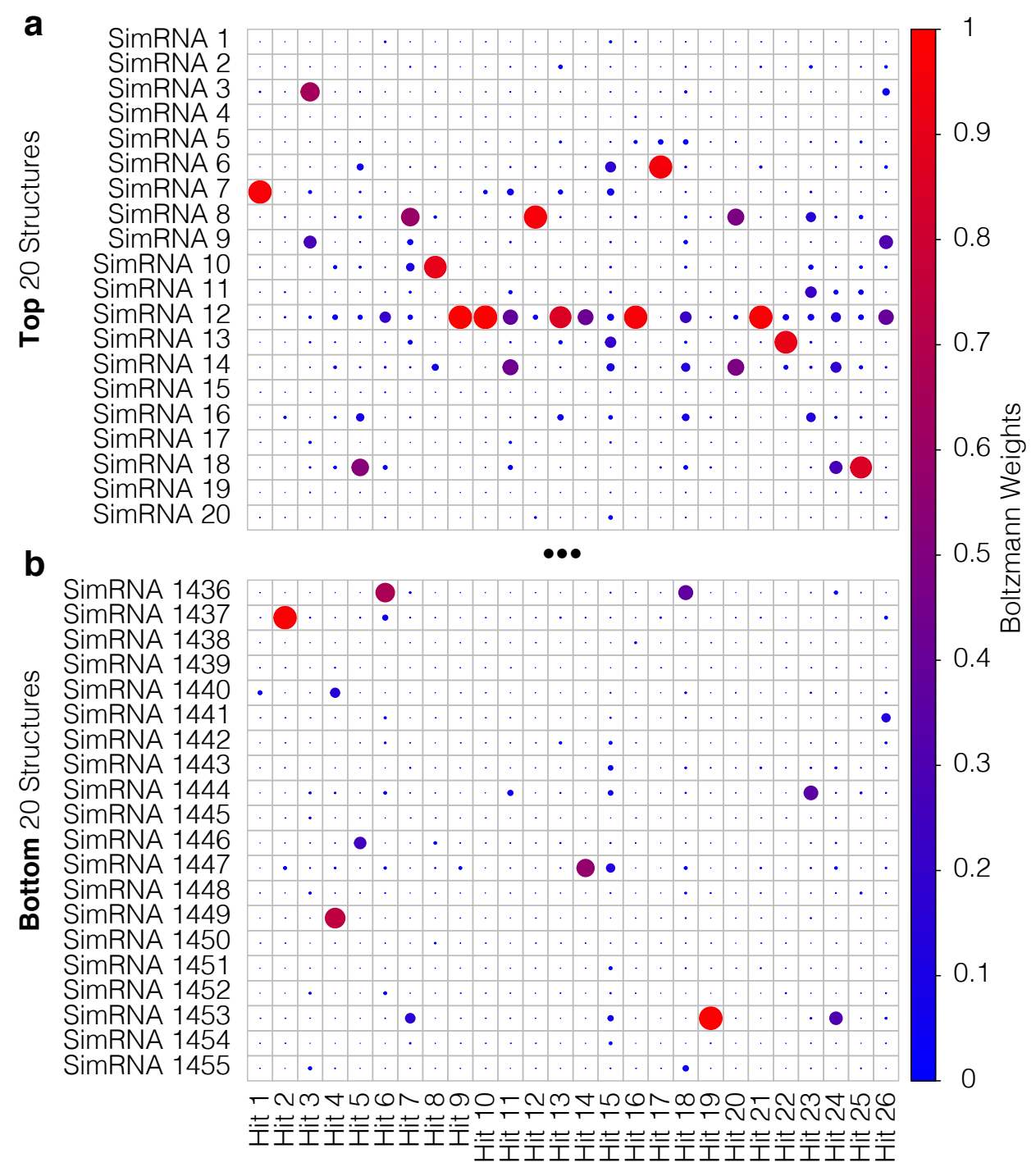

Figure S6. For comparison, we carried docking against the 20 SimRNA structures with the lowest cavity scores and compared the results with those obtained when we docked against the 20 structures with highest cavity scores. Shown here are the Boltzmann weights $\left(w_{i, j}=e^{-\Delta G_{i, j} / k_{B} T}\right)$ for the 26 hits docked against the 20 SimRNA TAR structures with (a) highest and (b) lowest cavity scores. The Boltzmann weights are higher when the hits are docked against the top 20 SimRNA structure relative to the bottom 20 structure. The sum of the Boltzmann weights, i.e., the partition function $\left(Q=\sum_{i} \sum_{j} w_{i, j}\right)$ was $\sim 19.5$ and $\sim 6.5$, for the top and bottom 20 SimRNA structures. Thus within an ensemble docking framework, the docking scores from the top 20 would contribute more to the over binding affinity, which is used to ranking compounds. When computing the Boltzmann weights $\left(w_{i, j}\right), \Delta G_{i, j}$ is the rDock interaction score for a ligand $i$ docked to conformer $j$, and $T$ was set to $298 \mathrm{~K}$.

\section{References}

1. Ruiz-Carmona, S., Alvarez-Garcia, D., Foloppe, N., Garmendia-Doval, A. B., Juhos, S., Schmidtke, P., Barril, X., Hubbard, R. E. \& Morley, S. D. rDock: a fast, versatile and open 
source program for docking ligands to proteins and nucleic acids. PLoS computational biology 10, e1003571 (2014).

2. Ravindranath, P. A. \& Sanner, M. F. Autosite: an automated approach for pseudo-ligands prediction-from ligand-binding sites identification to predicting key ligand atoms. Bioinforma. 32, 3142-3149 (2016).

3. Landrum, G. Rdkit: A software suite for cheminformatics, computational chemistry, and predictive modeling (2013).

4. Aboul-ela, F., Karn, J. \& Varani, G. The structure of the human immunodeficiency virus type-1 TAR RNA reveals principles of RNA recognition by Tat protein. J. molecular biology 253, 313-332 (1995).

5. Faber, C., Sticht, H., Schweimer, K. \& Rösch, P. Structural rearrangements of HIV-1 Tatresponsive RNA upon binding of neomycin b. J. Biological Chem. 275, 20660-20666 (2000).

6. Davis, B., Afshar, M., Varani, G., Murchie, A., Karn, J., Lentzen, G., Drysdale, M., Bower, J., Potter, A., Starkey, I., Swarbrick, T. \& Aboul-ela, F. Rational design of inhibitors of HIV-1 TAR RNA through the stabilisation of electrostatic "hot spots". J. molecular biology 336, 343-356 (2004).

7. Davidson, A., Begley, D. W., Lau, C. \& Varani, G. A small-molecule probe induces a conformation in HIV TAR RNA capable of binding drug-like fragments. J. molecular biology 410, 984-996 (2011).

8. Davidson, A., Leeper, T. C., Athanassiou, Z., Patora-Komisarska, K., Karn, J., Robinson, J. A. \& Varani, G. Simultaneous recognition of HIV-1 TAR RNA bulge and loop sequences by cyclic peptide mimics of Tat protein. Proc. Natl. Acad. Sci. 106, 11931-11936 (2009).

9. Davidson, A., Patora-Komisarska, K., Robinson, J. A. \& Varani, G. Essential structural requirements for specific recognition of HIV TAR RNA by peptide mimetics of Tat protein. Nucleic acids research 39, 248-256 (2011).

10. Borkar, A. N., Bardaro, M. F., Camilloni, C., Aprile, F. A., Varani, G. \& Vendruscolo, M. Structure of a low-population binding intermediate in protein-RNA recognition. Proc. Natl. Acad. Sci. 113, 7171-7176 (2016).

11. Belashov, I. A., Crawford, D. W., Cavender, C. E., Dai, P., Beardslee, P. C., Mathews, D. H., Pentelute, B. L., McNaughton, B. R. \& Wedekind, J. E. Structure of HIV TAR in complex with a lab-evolved RRM provides insight into duplex RNA recognition and synthesis of a constrained peptide that impairs transcription. Nucleic acids research 46, 6401-6415 (2018).

12. Boniecki, M. J., Lach, G., Dawson, W. K., Tomala, K., Lukasz, P., Soltysinski, T., Rother, K. M. \& Bujnicki, J. M. SimRNA: a coarse-grained method for RNA folding simulations and $3 d$ structure prediction. Nucleic acids research 44, e63-e63 (2015).

13. Aboul-ela, F., Karn, J. \& Varani, G. Structure of HIV-1 TAR RNA in the absence of ligands reveals a novel conformation of the trinucleotide bulge. Nucleic acids research 24, 39743981 (1996). 
14. Brooks, B. R., Bruccoleri, R. E., Olafson, B. D., States, D. J., Swaminathan, S. \& Karplus, M. CHARMM: a program for macromolecular energy, minimization, and dynamics calculations. J. computational chemistry 4, 187-217 (1983).

15. Denning, E. J., Priyakumar, U. D., Nilsson, L. \& Mackerell Jr, A. D. Impact of 2-hydroxyl sampling on the conformational properties of RNA: Update of the CHARMM all-atom additive force field for RNA. J. computational chemistry 32, 1929-1943 (2011).

16. Zok, T., Antczak, M., Zurkowski, M., Popenda, M., Blazewicz, J., Adamiak, R. W. \& Szachniuk, M. Rnapdbee 2.0: multifunctional tool for rna structure annotation. Nucleic acids research 46, W30-W35 (2018).

17. Ganser, L. R., Lee, J., Rangadurai, A., Merriman, D. K., Kelly, M. L., Kansal, A. D., Sathyamoorthy, B. \& Al-Hashimi, H. M. High-performance virtual screening by targeting a high-resolution RNA dynamic ensemble. Nat. structural \& molecular biology 25, 425-434 (2018). 